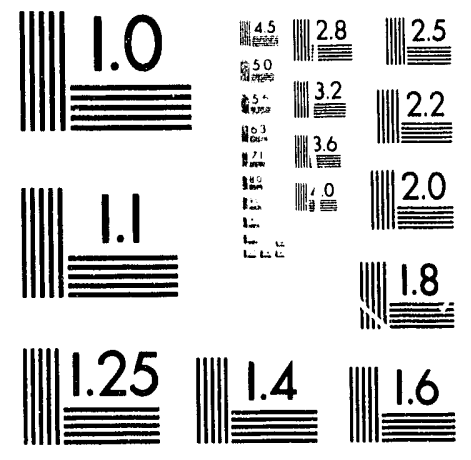



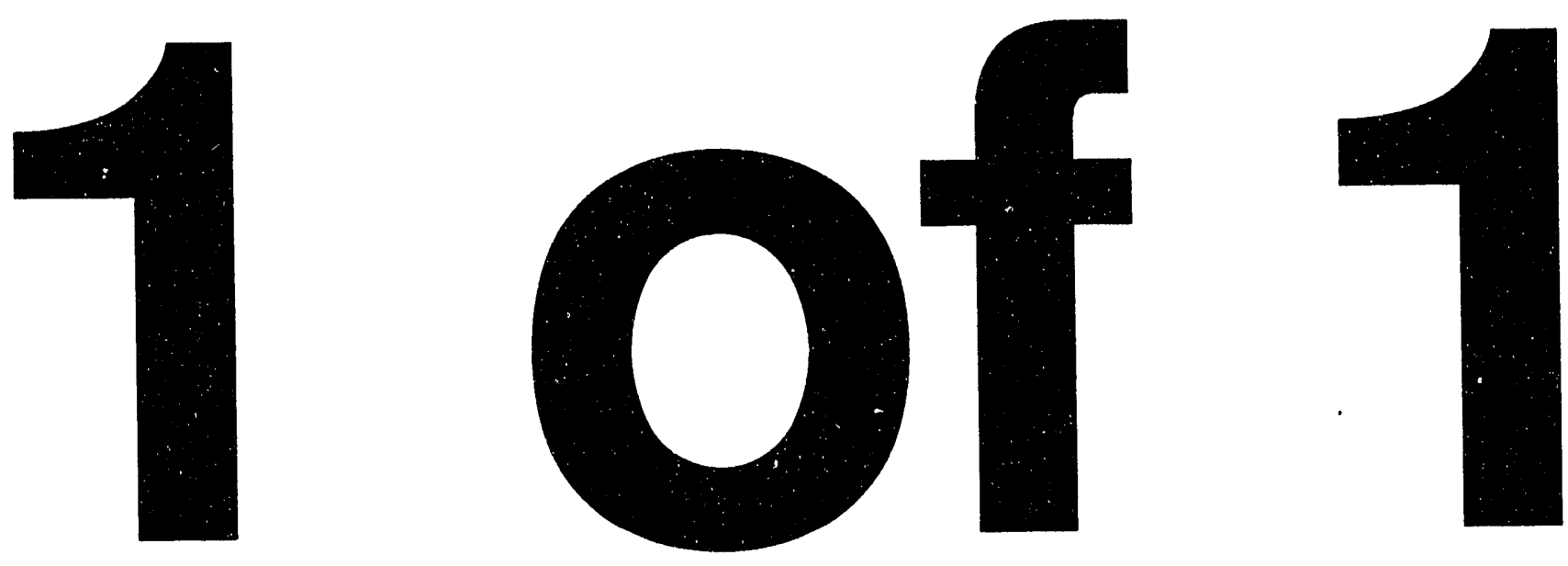


\title{
STUDIES OF DEEP LEVELS IN HIGH RESISTIVITY SILICON DETECTORS IRRADIATED BY HIGH FLUENCE FAST NEUTRONS USING A THERMALLY STIMULATED CURRENT SPECTROMETER*
}

\author{
Zheng Li, H. W. Kraner, W. Chen, R. Beuttenmuller \\ Brookhaven National Laboratory, Upton, NY 1197.3 USA \\ U. Biggeri, M. Bruzzi, E. Borchi, A. Baldini, and P. Spillantini \\ I.N.F.N. and University of Florence, Firenza, Italy
}

April 1993

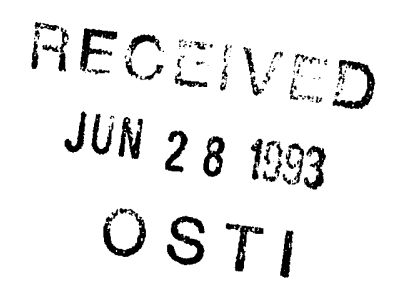

*This research was supported hy the U.S. Department of Energy: Contract No. I)E-AC()2-76CH()(0)16. 


\title{
Studies of Deep Levels in High Resistivity Silicon Dectectors Irradiated by \\ High Fluence Fast Neutrons Using a Thermally Stimulated Current Spectrometer
}

\author{
Z. Li, U. Biggeri*, M. Bruzzi*, E. Borchi*, \\ H. W. Kraner, W. Chen, R. Beuttenmuller, \\ A. Baldini*, and P. Spillantini* \\ Brookhaven National Laboratory \\ Upton, NY 11973 USA
}

*I.N.F.N. and University of Florence,

Firenza, Italy

Measurements of deep level spectrum of high resistivity silicon detectors irradiated by high fluence fast neutrons $\left(\Phi_{\mathrm{n}}: 2 \times 10^{12} \mathrm{n} / \mathrm{cm}^{2}\right.$ to $\left.1 \times 10^{14} \mathrm{n} / \mathrm{cm}^{2}\right)$ have been made using a thernally stimulated current (TSC) spectrometer. It has been found that at least nine new defect levels, with peaking temperature of $19 \mathrm{~K}$, $27 \mathrm{~K}, 36 \mathrm{~K}, 44 \mathrm{~K}, 49 \mathrm{~K}, 83 \mathrm{~K}, 93 \mathrm{~K}, 105 \mathrm{~K}$, and $120 \mathrm{~K}$, begin to appear when $\Phi_{\mathrm{n}} \geq$ $1 \times 10^{13} \mathrm{n} / \mathrm{cm}^{2}$. All peaks have strong dependences on the filling voltage $\left(V_{\text {tily }}\right.$, forward bias) or injection current especially for high fluence $\left(\Phi_{\mathrm{n}} \geq 10^{13} \mathrm{n} / \mathrm{cm}^{2}\right)$ situations. The defect concentration, energy level in the band gap, and cross section of each deep level, totaling at least 13 , have been studied systematically and possible identifications of the levels have been discussed.

\author{
Zheng Li \\ Brookhaven National Laboratory \\ Instrumentation \\ Building 535B \\ Upton, NY 11973, USA \\ (516) $282-5773$ \\ ZhengL@BNLCL1
}

\section{DISCLAIMER}

\begin{abstract}
This report was prepared as an account of work sponsored by an agency of the United States Government. Neither the United States Government nor any agency thereof, nor any of their employees, makes any warranty, express or implied, or assumes any legal liability or responsibility for the accuracy, completeness, or usefulness of any information, apparatus, product, or process disclosed, or represents that its use would not infringe privately owned rights. Reference herein to any specific commercial product, process, or service by trade name, trademark, manufacturer, or otherwise does not necessarily constitute or imply its endorsement, recommendation, or favoring by the United States Government or any agency thereof. The views and opinions of authors expressed herein do not necessarily state or reflect those of the United States Government or any agency thereof.
\end{abstract}




\section{SUMMARY}

Physical parameters of displacement radiation induced defect levels in silicon, such as defect energy levels in the band gap $E_{t}$, carrier capture cross sections $\sigma_{t}$, and defect concentration $N_{t}$, have been traditionally studies by the capacitive deept level transient spectroscopy (DLTS) for low resistivity silicon ( $\rho \leq 10 \Omega$-cm) or $N_{d} \geq-10^{14} / \mathrm{cm}^{3}$ ), for which the requirements for valid DLTS results, $\mathrm{N}_{\mathrm{t}} / \mathrm{N}_{\mathrm{d}}<<1$, is readily satisfied $[1-5]$. Althoguh recently DLTS has also been applied to neutron radiation induced defact levels in high resistivity silicon $\left(\rho \geq 2 \mathrm{~K} \Omega-\mathrm{cm}\right.$, or $\left.\mathrm{N}_{\mathrm{d}}<1 \times 10^{12} / \mathrm{cm}^{3}\right)$, its use has been largely limited to samples with low neutron fluences $\left(\Phi_{\mathrm{n}}<10^{12} / \mathrm{cm}^{2}\right)$ to keep the defect concentration low $\left(\mathrm{N}_{\mathrm{t}} \leq 10^{12} / \mathrm{cm}^{3}\right)^{[6-9]}$. Other techniques that use current or current transient instead of capacitance transient, such as laser-DLTS[10] and thermally stimulated current (TSC) $[11-14]$, have been proven not limited by the ratio of $\mathrm{N}_{\mathrm{t}} / \mathrm{N}_{\mathrm{d}}$ and good analytical tools for studying defect levels in high resistivity silicon (or other semiconductors) irradiated by high fluence fast neutrons or other particles that cause desplacement damage. In this paper, we will report new defect levels in heavily neutron irradiated high resistivity silicon detectors revealed by the TSC technique.

Silicon $\mathrm{p}^{+}-\mathrm{n}^{-}-\mathrm{n}^{+}$junction detectors used in this study were made on $\mathrm{n}$-type $\langle 111\rangle$ wafers with resistivity of $4-6 \mathrm{~K} \Omega-\mathrm{cm}$ in Brookhaven National Laboratory's Silicon Detector Development and Processing Lah (SDDPL). Fast neutrons form $10 \mathrm{keV}$ to 2.2 $\mathrm{MeV}$, with an average energy of $1 \mathrm{MeV}$, were obtained from the ${ }^{7} \mathrm{Li}(\mathrm{p}, \mathrm{n})$ reaction using 4 $\mathrm{MeV}$ protons from a Van de Graaff accelerator at the University of Lowell. Detectors from the same wafer, with the same processing conditions, were irradiated to various neutron fluences ranging from $2 \times 10^{12} \mathrm{n} / \mathrm{cm}^{2}$ to about $1 \times 10^{14} \mathrm{n} / \mathrm{cm}^{2}$, and were measured with the TSC set-up at the University of Florence, Italy.

The TSC spectrum of a detector without any radiation is shown in Fig. 1a. Only two peaks, $T=17 \mathrm{~K}$ and $\mathrm{T}=25 \mathrm{~K}$, have been found for this undamaged detector. The peaks at $T=17 \mathrm{~K}$ and $t=25 \mathrm{~K}$ are very shallow $\left(E_{t}\right.$ in the order of $<100 \mathrm{meV}$ ), and are probably doping impurities phosphrous and boron, respectively. Fig. Ib shows the TSC spectrum of a detector irradiated to $4.79 \times 10^{12} \mathrm{n} / \mathrm{cm}^{2}$. Note that the peak at $17 \mathrm{~K}$ becomes much narrower and the peak at $25 \mathrm{~K}$ smaller, indicating deactivation of both type of doping impurities or carrier removal. Also, two new peaks, one at $19 \mathrm{k}$ and one between 140 to $170 \mathrm{k}$, denoted as the "C" peak, begin to take shape. The broad peak between $140 \mathrm{~K}$ to 170K was studied extensively by Bruzzi. et. al, and was attributed as a cluster[12].

Many new peaks between $T=20 \mathrm{~K}$ to $130 \mathrm{~K}$ start to appear when the fluence is in the order of $1 \times 101)^{13} \mathrm{n} / \mathrm{cm}^{2}$, as shown in Fig. 2 a. these new peaks take clear shapes when 
the fluence is about $3-4 \times 10^{13} \mathrm{n} / \mathrm{cm}^{2}$, while the borad " $\mathrm{C}$ " peak continues to grow wider and higher, as shown in Fig. $2 \mathrm{~b}$. The peaking temperatuires for the new peaks are $19 \mathrm{~K}$, $27 \mathrm{~K}, 30 \mathrm{~K}, 44 \mathrm{~K}, 49 \mathrm{~K} 83 \mathrm{~K}, 93 \mathrm{~K}, 105 \mathrm{~K}$, and $120 \mathrm{~K}$. The peak at $\mathrm{T}=70 \mathrm{~K}$, denoted as "A" peak, also appears at lower fluences and is the oxygen-vacancy (A-center) complex. With further radiation of neutrons, as it is shown in fig. 3, some of these new peaks simply become higher, others overlap and become broad peaks. We note that there are little changes for peaks at $17 \mathrm{~K}$ and $25 \mathrm{~K}$ when $\Phi_{\mathrm{n}}>5 \times 10^{12} \mathrm{n} / \mathrm{cm}^{2}$, This fluence value is consistent with the one at which the type inversion in the space-charge-region (SCR) was observed [15-17].

We must note here that all peaks in Fig. I a to Fig. 3 were obtained, or observable, only in conditions of high positive filling voltage $V_{\text {fill }}$, or more appropriate, the high injection currents ( $>\mu \mathrm{A}$ 's) during the filling process. Without high injection current, nothing could be seen. This is demonstrated in Fig. 4 a (no peaks when $\mathrm{I}_{\text {inj }}$ was low) and Fig. $4 b$ (all peaks when $I_{\text {inj }}$ was high). The $V_{\text {fill }}$ dependence of the " $C$ " peak of a heavily damaged detector was shown in Fig. 5a and 5b, which agrees with the early work of Bruzzi, et. al, [12]. The $V_{\text {fill }}$ dependence of the "A" peak of a lesser damaged detector was plotted in Fig. 6. As the defect concentration increases with neutron fluences, one needs to inject certain amounts of carriers into the detector to fill the levels. Since for a given injection current, the forward bias becomes larger as neutron fluence increases [18], the $\mathrm{V}_{\text {fill }}$ dependence of peaks other than the " $\mathrm{C}$ " peak (for which the $\mathrm{V}_{\text {fill }}$ dependence was explained as charge trapping of a cluster [12]) may be just a reflection of the $I_{\text {inj }}$ requirement. More data and detailed modeling of $\mathrm{V}_{\text {fill }}$ dependence will be published elsewhere[19].

The physical paramerers $\left(E_{t}, \sigma_{t}, N_{t}\right)$ of each peak can be obtained by analyzing the deexcitation data shown in Fig. 7. By waiting for various times $\left(t_{d}\right)$ before the TSC run, one can get a decay data of each peak from which the parameters can be obtained. In the full paper, the physical parameters of each peak, and their dependence on the neutron fluence, will be reported. 


\section{REFERENCES}

1. L. C. Kimerling, "Defect States in Electron-hombarded Silicon: Capacitance Transient analyses," in: Radiation Effects in Semiconductors, (The Institute of Physics, Bristol, UK), 1976 Institute of Physics Conf. Series $\underline{31}, 221-230$ (1977).

2. Y. Tokuda and a. Usami, "Comparison of Neutron and $2 \mathrm{MeV}$ Electron Damage in n-type Silicon by Deep-level Transient Spectroscopy," IEEE Trans. Nucl. Sci. NS-28(3), 3564-3568 (1981).

3. Y. Tokuda and A. Usami, "DLTS Studies of Neutron Damage in p-type Silicon," IEEE Trans. Nucl. Sci. NS-29(5), 1388-1392 (1982).

4. S. D. Brotherton and P. Bradley, "Defect Production and Lifetime Control in Electron and $\gamma$ irradiated Silicon," J. Appl Phys. 53(8), 5720(1982.

5. O. O. Awadelkarim and B. Monemar, "Defect States in 2.0-MeV Electron Irradiated PhosphorusDoped Silicon," J. Appl. Phys., 65, 4779-4788 (1989).

6. L. Vismara SICAPO Collaboration: Radiation Damage Effects on Silicon Detectors, presented at the Int. Conf. on Advanced Technology and Particle Physics. Comoo, Italy, June 13-16, 1988.

7. Zheng Li, W. Chen, and H. W. Kraner, "Effects of Fast Neutron Radiation on the electrical Properties of Silicon Detectors," Nucl. Instr. and Meth., A308, 585 (1991).

8. E. Borchi, C. Bertrand, C. Leroy, M. Bruzzi, C. Furetta, R. Paludetto, P. G. Rancoita. L. Vismara, and P. Giubellino, "Deep-level Transient Spectroscopy Measurements of Majority Carrier Traps in Neutron-Irradiated n-type Silicon Detectors." Nucl. Instrum. Methods, A279, 277-280 (1989).

9. Zheng Li, H. W. Kraner, e. Verbitskaya, V. Eremin, A. Ivanov, Monica Rattaggi, P. G. Rancoita, F. A. Rubinelli, S. J. Fonash, C. Dale, and P. Marshall, "Investigation of the Oxygen-Vacancy (Acenter) Defect Complex Profile in Neutron Irradiated High Resistivity Silicon Junction Particle Detectors," IEEE Trans. Nucl. Sci., Pt I, 1730 (1992).

10. Chengji Li and Zheng Li, "Characterizations of High Fluence Neutron Induced Defect Levels in High Resistivity Silicon Detectors Using a Laser-Deep Level Transient Spectroscope (L-DLTS)," to be presented at the International Symposium on Development and Application of Semiconductor Tracking Detectors, Hiroshima, Japan, May 22-24, 1993, to be published in Nacl. Instr. and Meth.

11. H. M. Heijne, J. C. Muller and P. Siffert, "TSC Defect Level in Silicon Produced by Irradiation with Muons of GeV Energy," Rad. Effects, 29, 25 (1976).

12. M. Bruzzi, E. Borchi, and A. Baldini, "Using Thernally Stimulated Currents to Visualize Defect Cluster in Neutron Irradiated Silicon," J. Appl. Phys., 72(9), 4007 (1992).

13. E. Borchi, M. Bruzzi, and M. S. Mazzoni. "Thernally Stimulated and Leakage Current Analysis of Neutron Irradiated Silicon Detectors," Nucl. Instr. and Meth., A310, 273 (1991).

14. A. Baddini, E. Borchi, M. Bruzzi, and P. Spillantini, "Electrical and Spectroscopic Analysis of Neutron-Irradiated Silicon Detectors," Nucl. Instr. and Meth., A315, 182 (1992).

15. G. Lindstrom, M. Benkert, E. Fretwurst, T. Schulz and R. Winston, Proc, of the First International Cont. on Calorimetry in High Energy Physics, D). F. Anderson, et al., Des, World Scientific Publishing (o., Singapore, 467 (190)). 
16. H. W. Kraner, Zheng Li, and E. Fretwurst, "the Use of the Signal Current Pulse Shape to Study the Internal Electrical Field Profile and Trapping Effects in Neutron Damaged Silicon Detectors," BNL47506, Nucl. Instr. and Meth., A326, 350 (1993).

17. Zheng Li, V. Eremin, N. Strokan, and E. Verbitskaya, Presented at the IEEE Nucl. Sci. Symp., Orlando, FL, Oct. 25-31, 1992. To be published in the IEEE Trans. Nucl. Sci. (in press).

18. Zheng Li, Unpublished data.

19. Zheng $\mathrm{Li}$, et al, to be published. 
TSC Spectrum - F $=0$

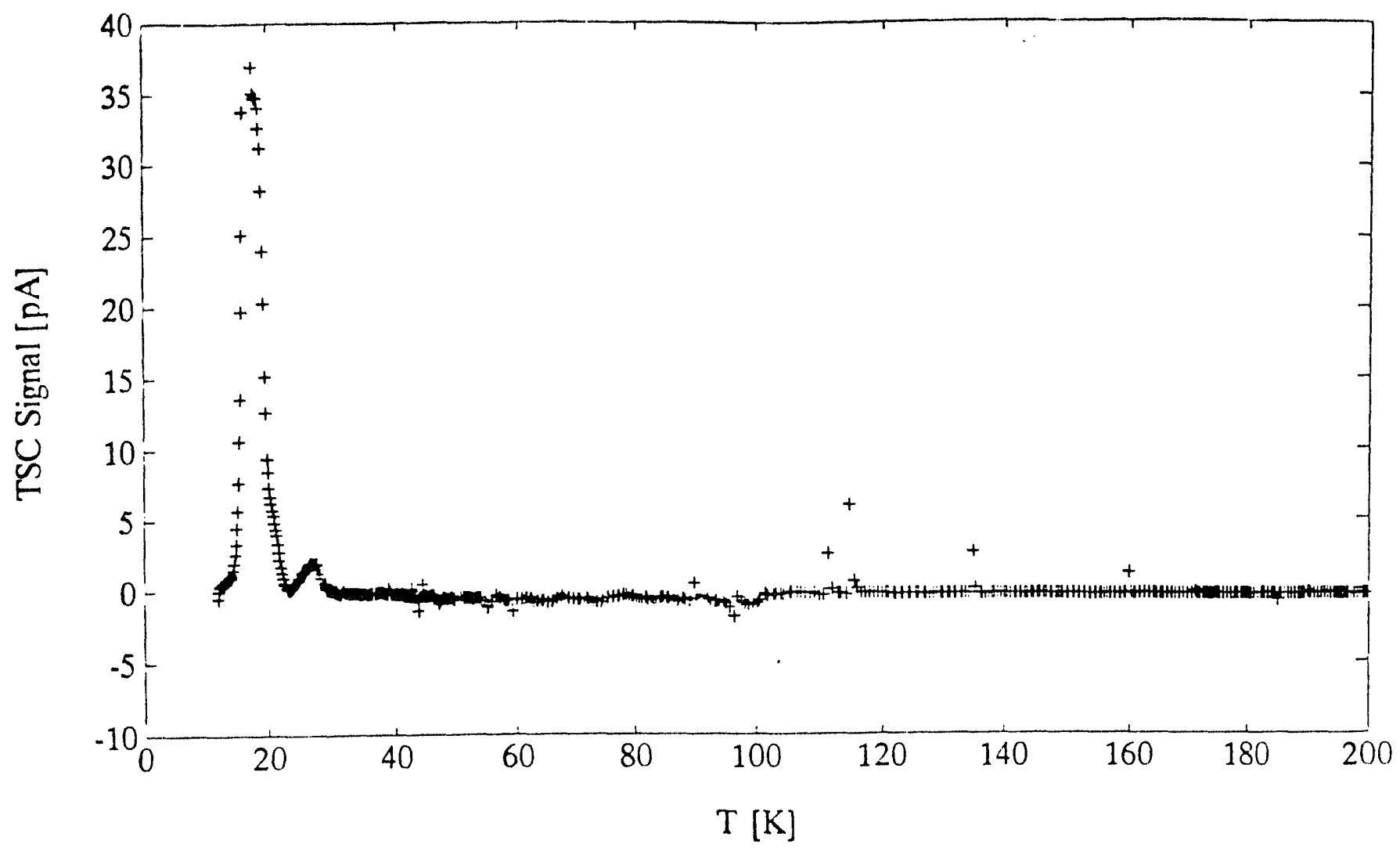

a) Not isradiated, $\Phi_{n}=0$

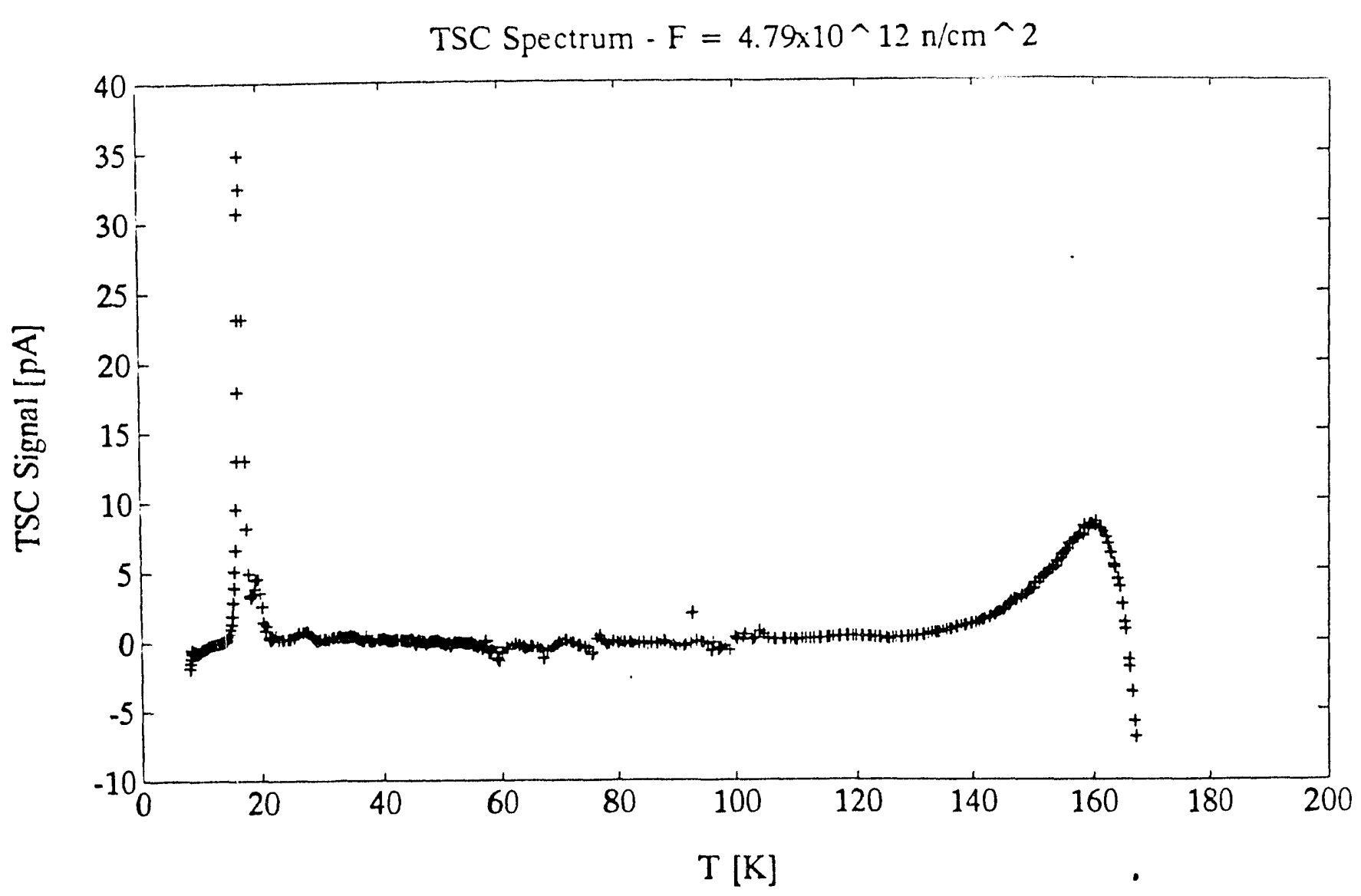

b) Irradiated to a medium fluence, $\Phi_{\mathrm{n}}=4.8 \times 10^{12} \mathrm{n} / \mathrm{cm}^{2}$

Fig. 1 TSC spectrum for a control detector and a defecenr irradialled to a medium fluence. 


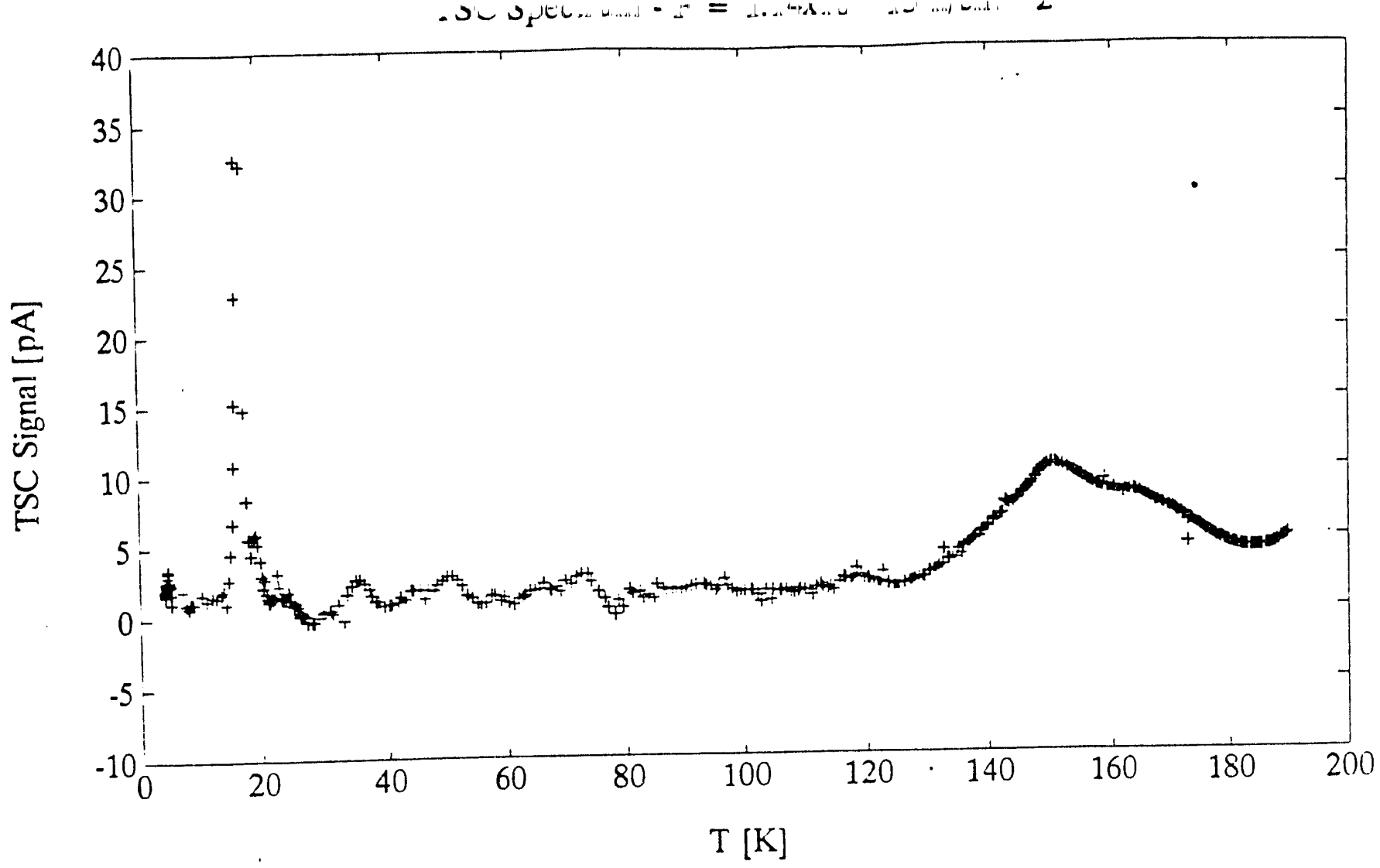

a) $\Phi_{n}=1.14 \times 10^{13} \mathrm{n} / \mathrm{cm}^{2}$

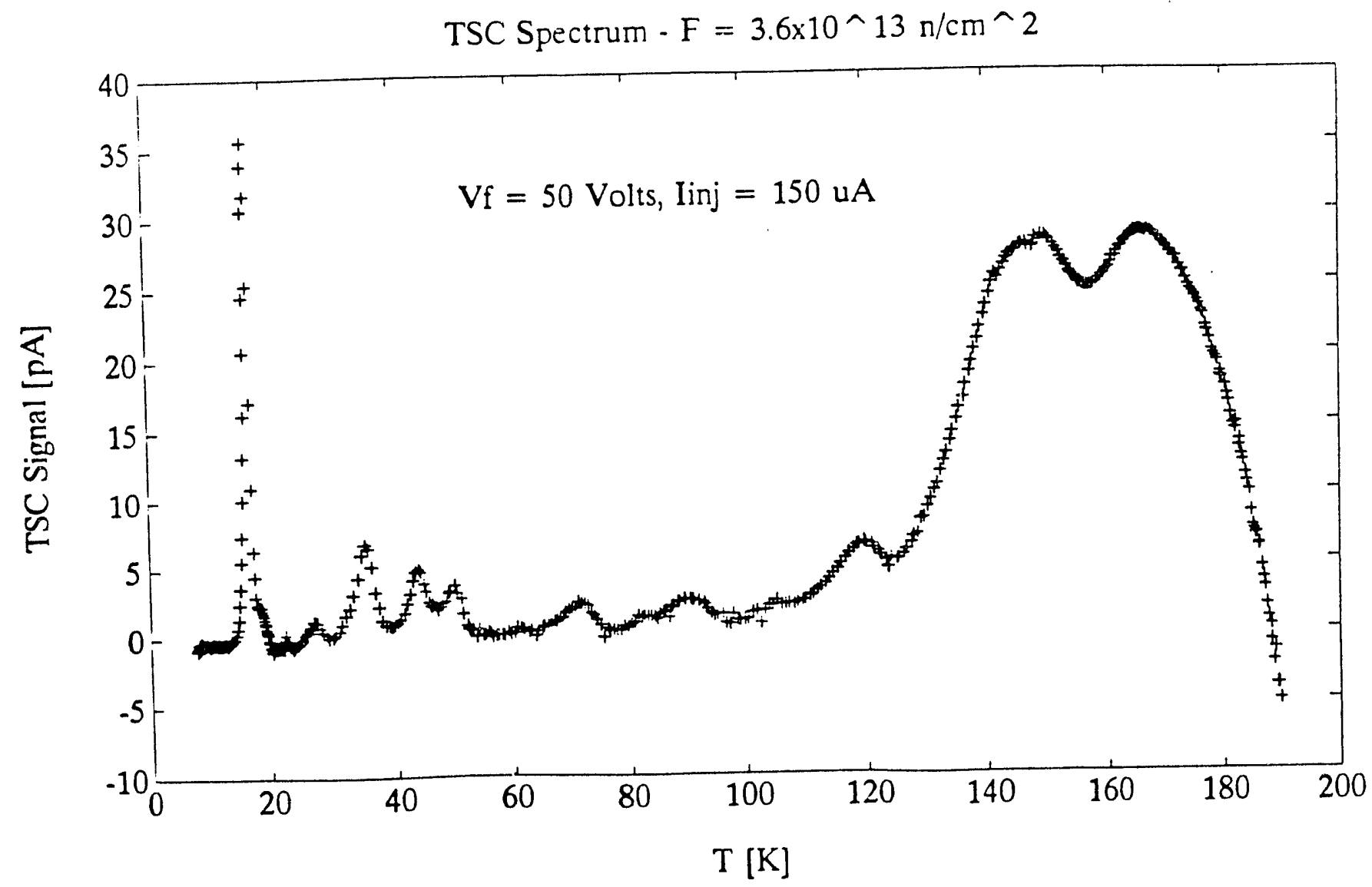

b) $\Phi_{n}=3.6 \times 10^{1.3}{ }_{n} / \mathrm{cm}^{2}$

Fig. 2 TSC spectrum for heavily irradiated detectors. 


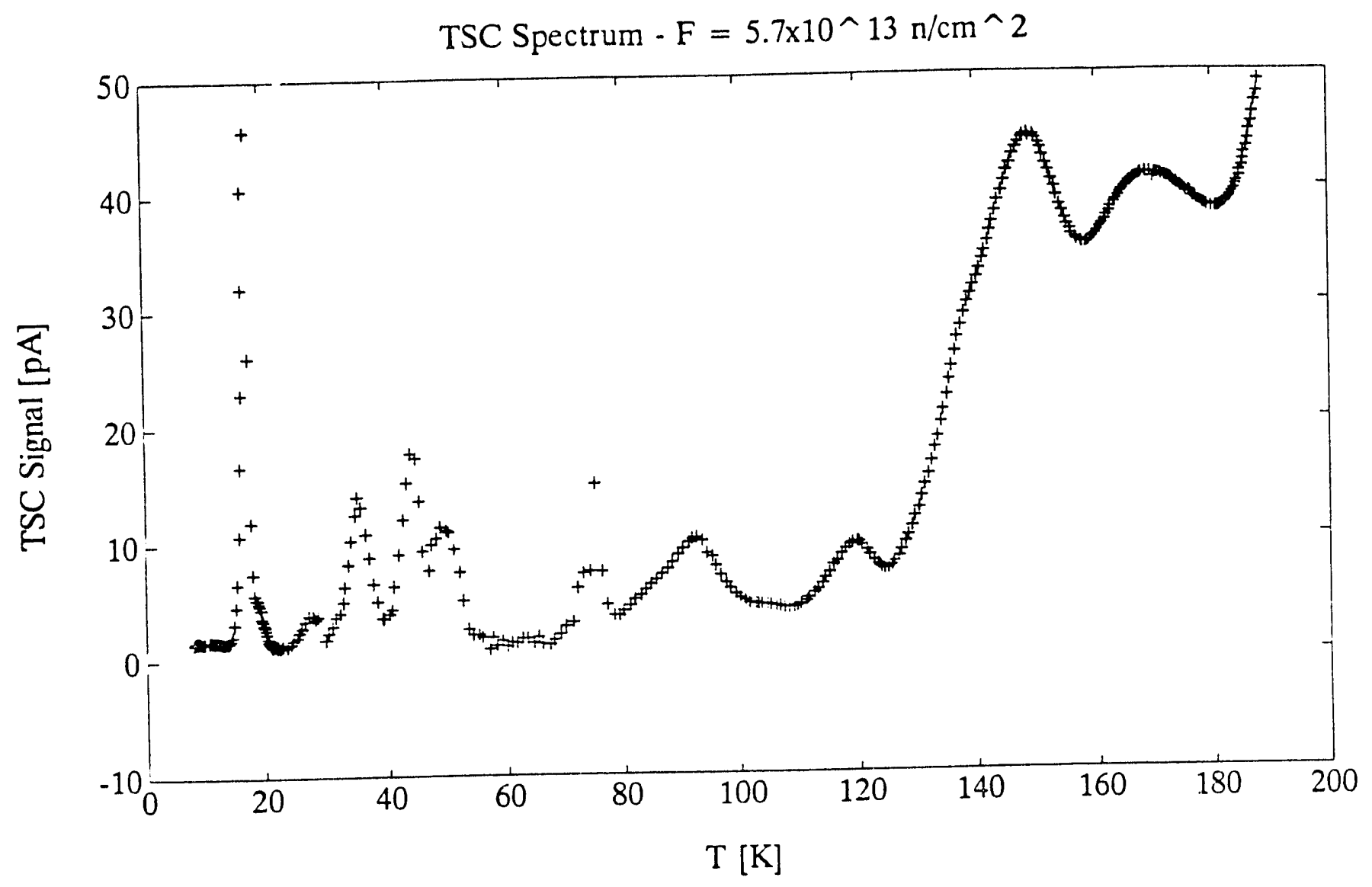

Fig. 3 TSC spectrum for a detector irradiated to $5.7 \times 10^{13} \mathrm{n} / \mathrm{cm}^{2}$ 


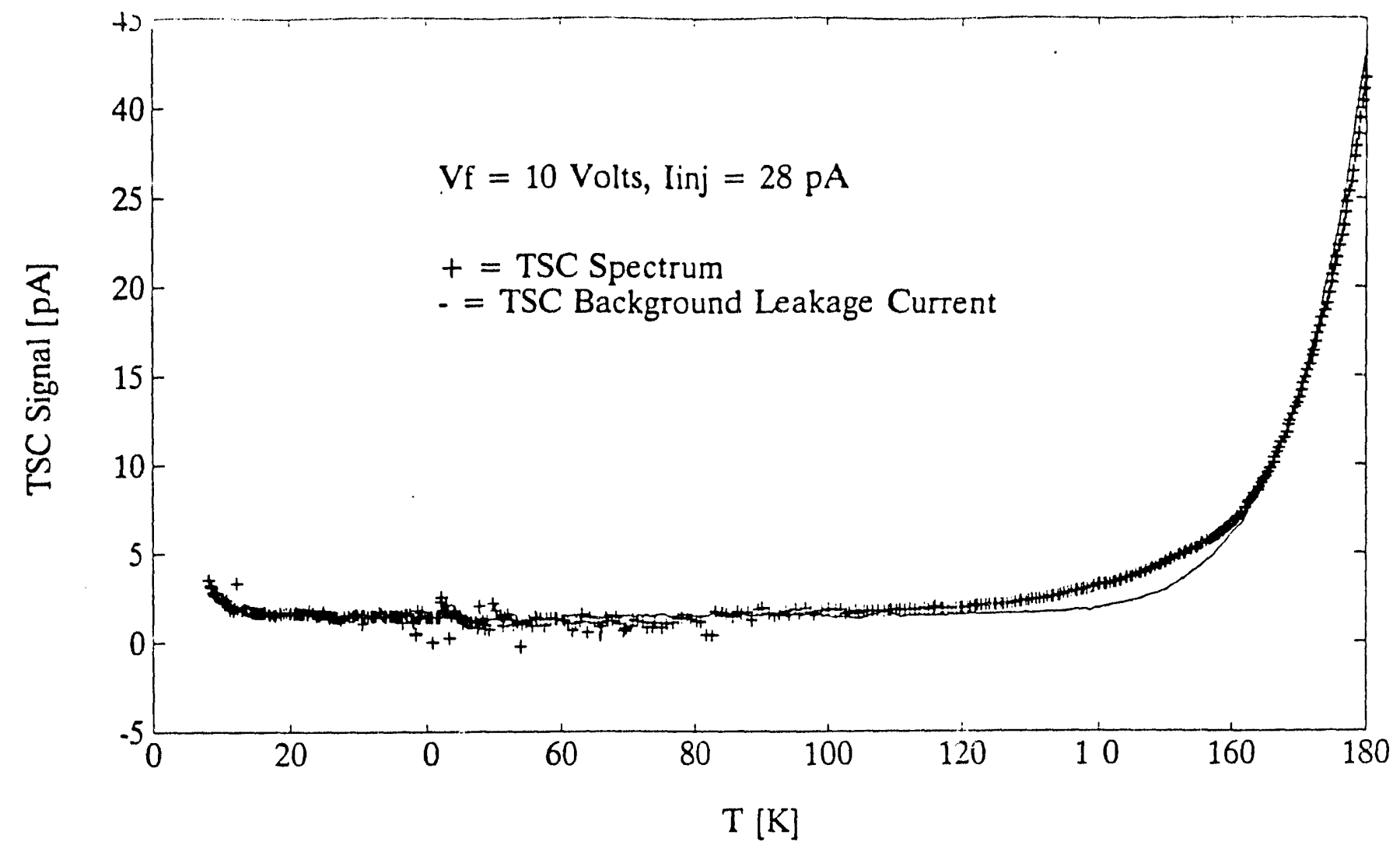

a) Low injection current.

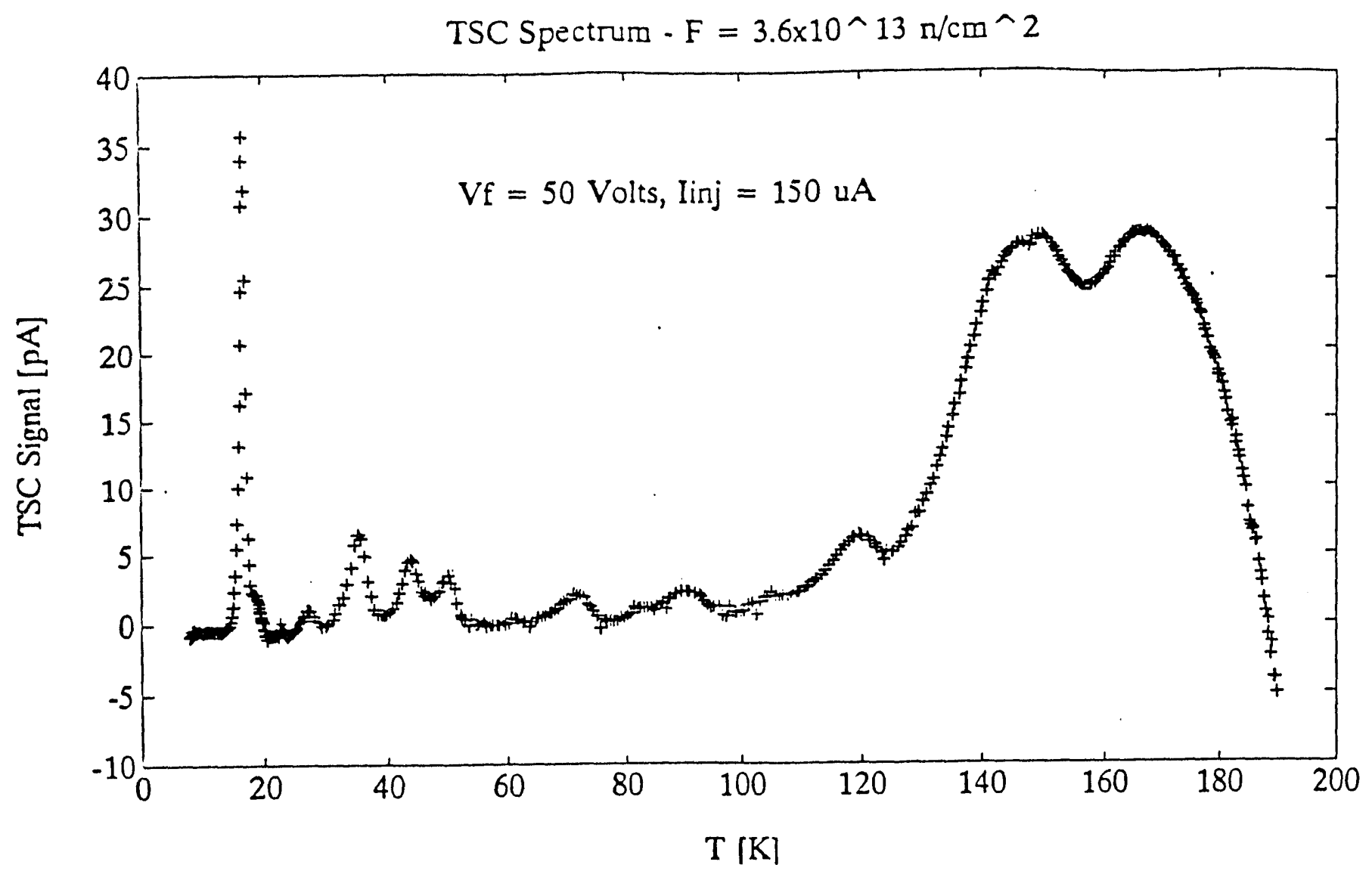

b) Iligh injection current.

Fig. 4 Filling vollage or injection current dependence of TSC peaks. 


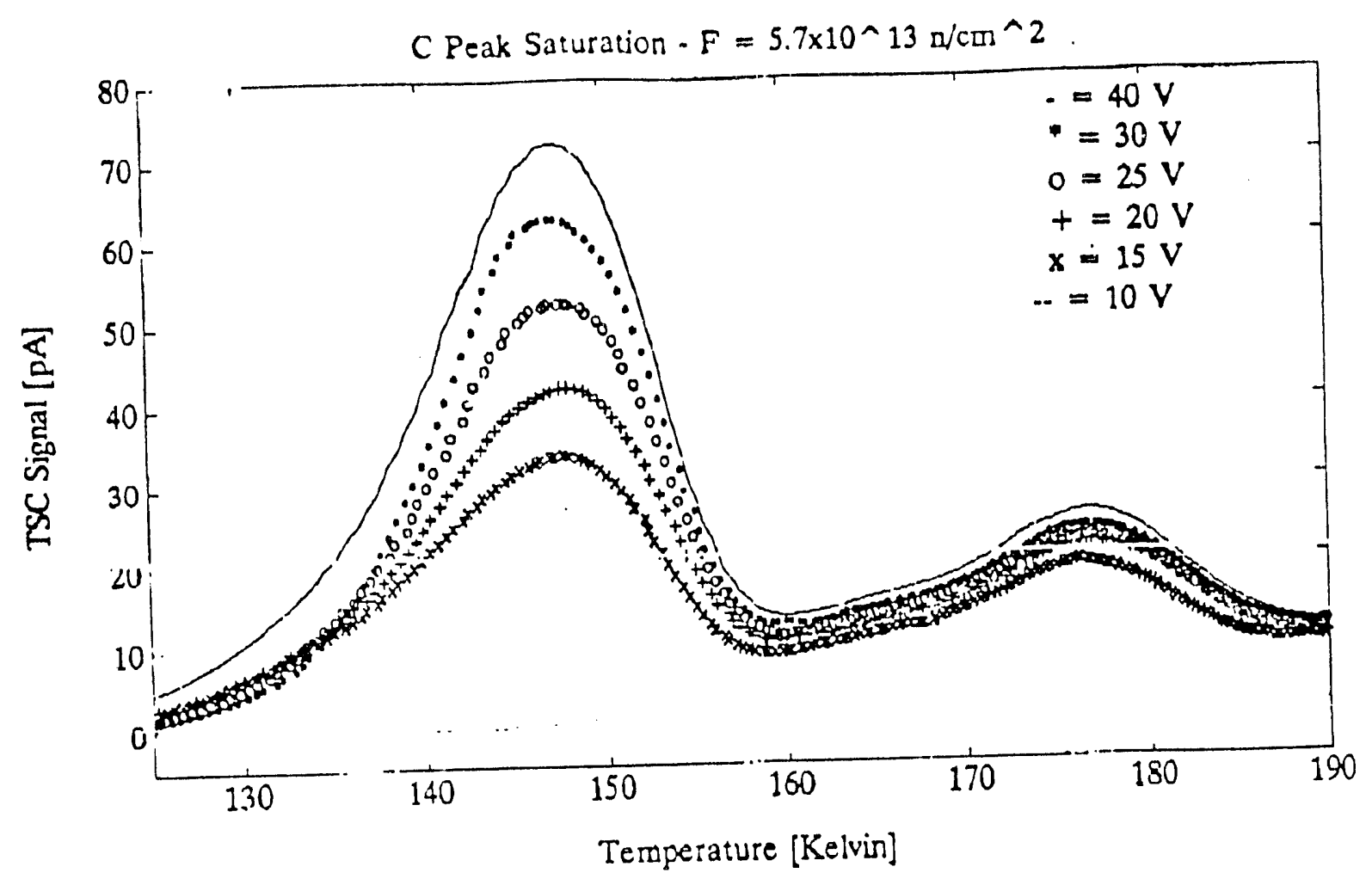

a) TSC spectrum.

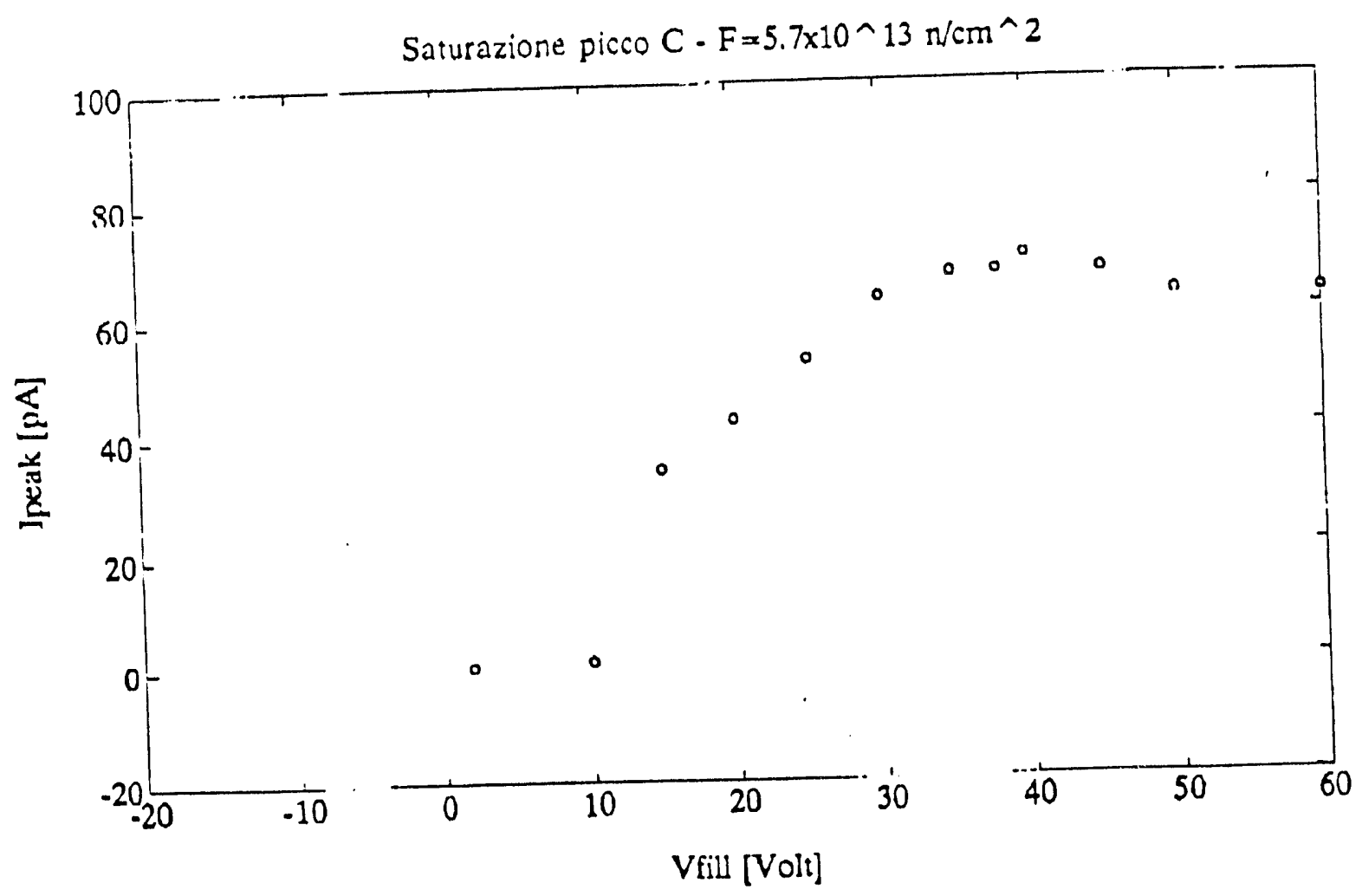

b) Peak high dependence.

Fig. 5 TSC "C" peak height dependence on the filling voltage. 


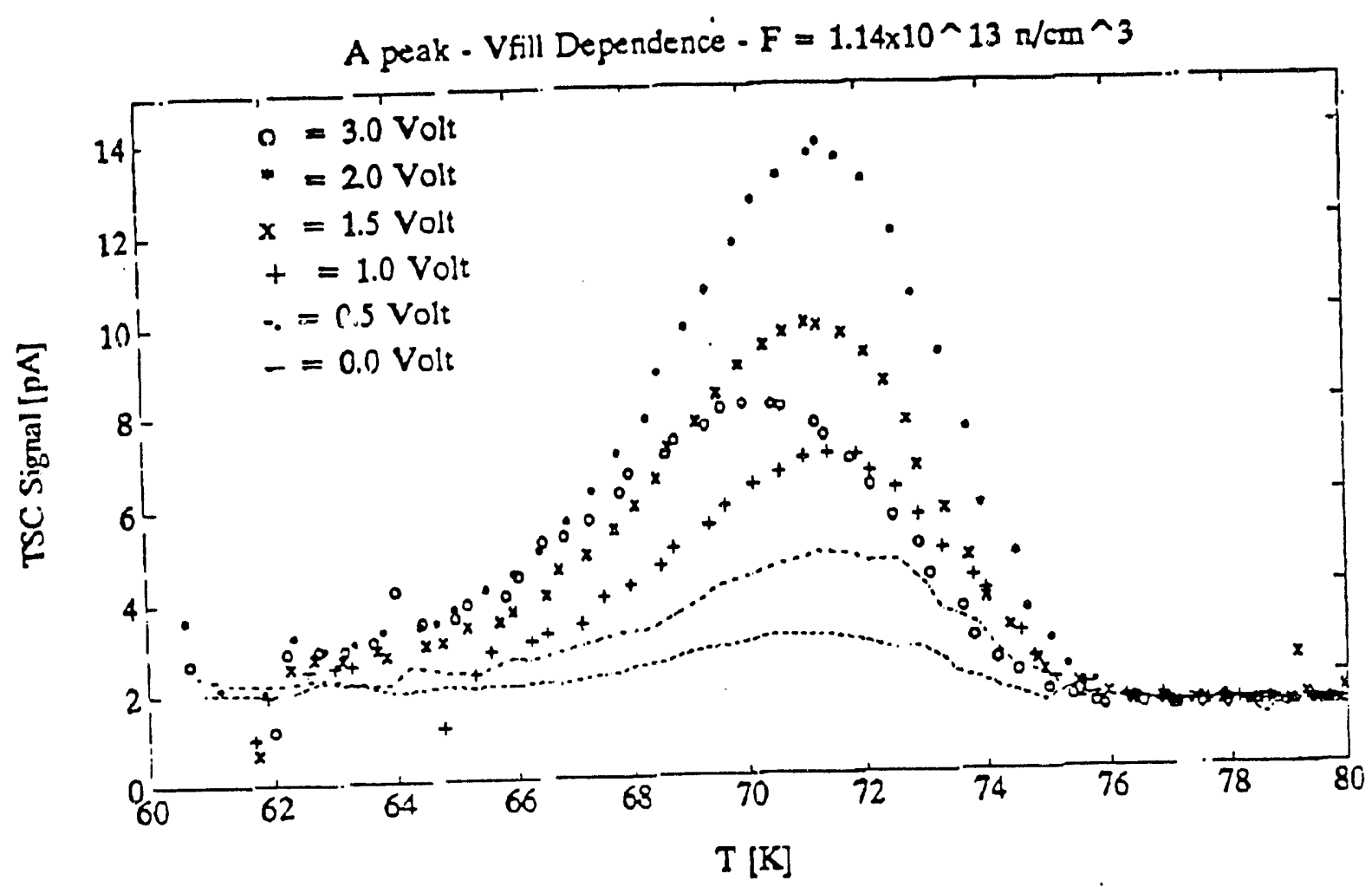

Fig. 6 TSC "A" peak height dkependence on the filling voltage. 


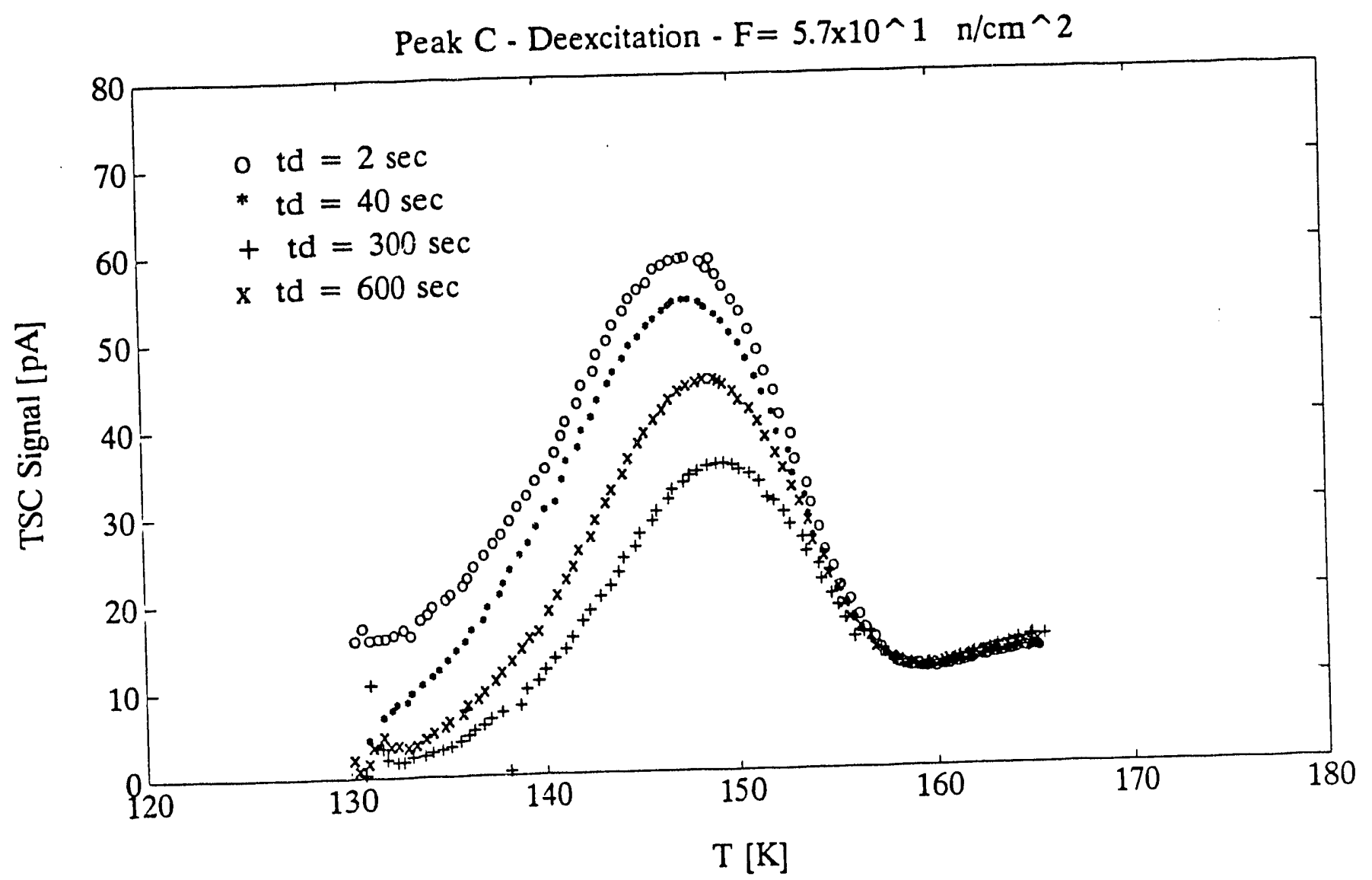

Fig. 7 TSC peak height dependence on the excitation time. 

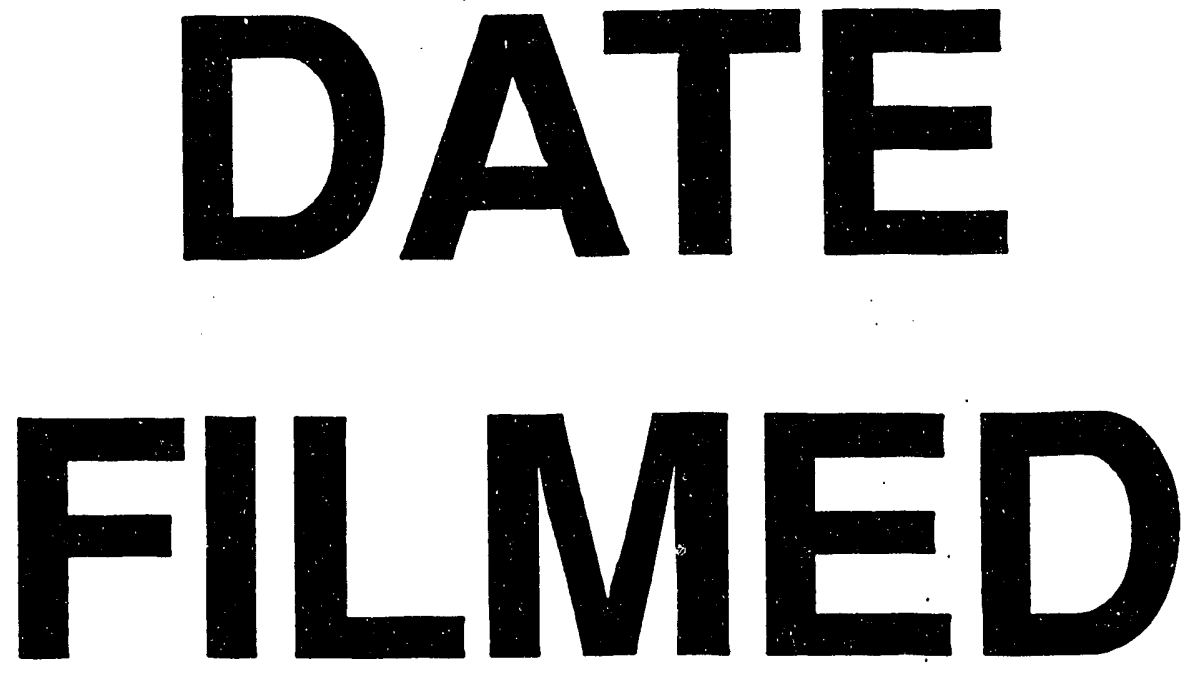

$10 / 13 / 93$
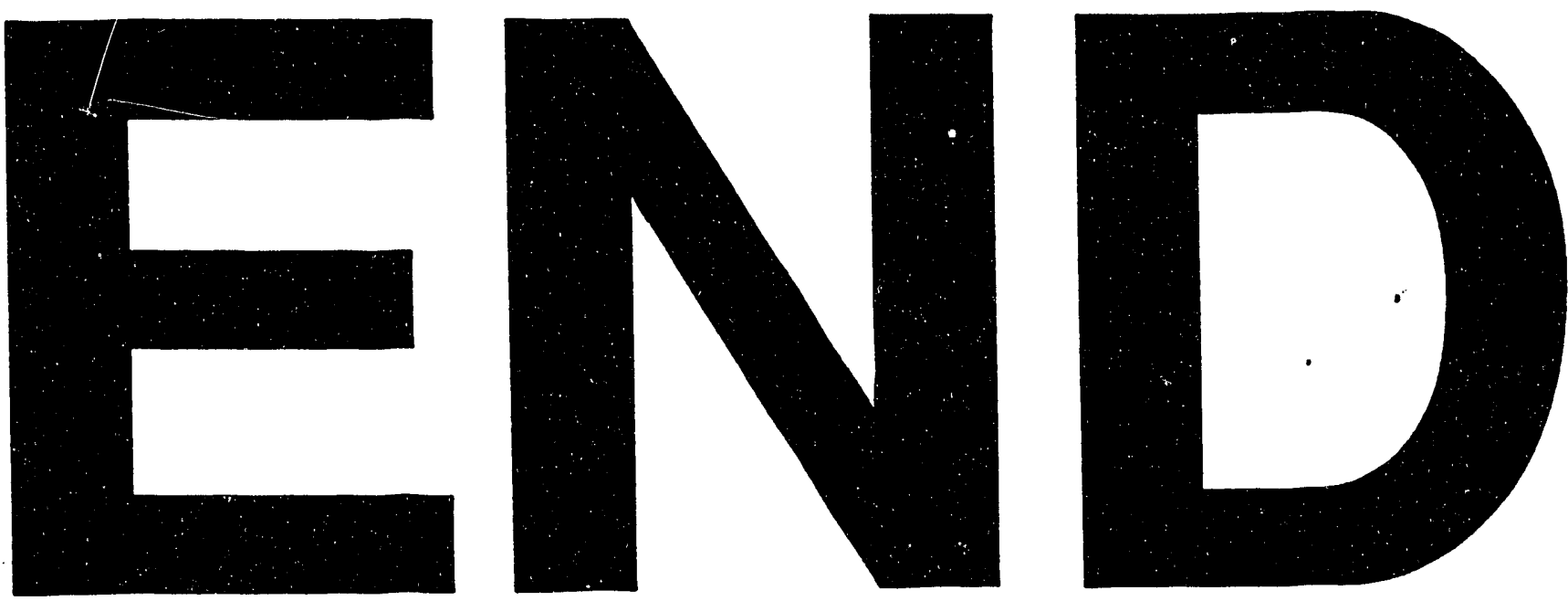
